INFLAMMATION

\title{
Glycoprotein (gp) 96 expression: induced during differentiation of intestinal macrophages but impaired in Crohn's disease
}

\author{
K Schreiter, M Hausmann, T Spoettl, U G Strauch, F Bataille, J Schoelmerich, H Herfarth, W Falk, \\ G Rogler
}

Gut 2005;54:935-943. doi: 10.1136/gut.2004.053116

See end of article for authors' affiliations

....................

Correspondence to: Dr G Rogler, Department of Internal Medicine I, University of Regensburg, 93042 Regensburg, Germany;

gerhard.rogler@

klinik.uni-regensburg.de

Revised version received 13 January 2005

Accepted for publication

16 February 2005 tive with the innate immune system. It is a chaperone Background: The glycoprotein (gp) 96 links the adaptive with the innate immune system. It is a chaperone
with a binding domain for peptides generated by proteasomal degradation. During cellular stress, peptide loaded gp96 can be released and presented to T cells by antigen presenting cells (APCs).

Methods: mRNAs from in vitro differentiated macrophages (iv mac) and normal intestinal macrophages (IMACs) were compared by subtractive hybridisation and Affymetrix GeneChip analysis. Differentiation induced expression of gp96 was investigated in the multicellular spheroid (MCS) model. In vivo gp96 protein expression was detected by double labelling immunohistochemistry of human colon and in the $\mathrm{CD} 4{ }^{+} \mathrm{CD} 62 \mathrm{~L}^{+} \mathrm{T}$ cell transfer mouse model.

Results: Five of 76 clones obtained by subtractive hybridisation revealed $>99 \%$ sequence homology to gp96. Affymetrix GeneChip analysis confirmed induction of gp96 in IMACs. Gp96 mRNA was detected in IMACs from normal and intestinal bowel disease mucosa. Induction of gp96 protein was observed after seven days in the MCS model of IMAC differentiation. Immunohistochemistry confirmed the presence of gp96 protein in IMACs in normal mucosa as well as in mucosa from patients with ulcerative colitis and diverticulitis. In mucosa from Crohn's disease (CD) patients, gp96 protein was not detectable. In the CD4 ${ }^{+}$ $\mathrm{CD} 2 \mathrm{~L}^{+} \mathrm{T}$ cell transfer mouse model, gp96 was verifiable in non-activated IMACs.

Conclusion: Gp96 is induced during differentiation of normal IMACs but is not detected in IMACs in CD mucosa. As gp96 has been described as having a role in tolerance induction, this may be relevant for loss of tolerance against luminal bacteria found in CD patients.
$\mathrm{T}$ he intestinal mucosa forms the primary barrier against the multitude of bacteria in the gut lumen. The immediate and effective recognition of bacterial products invading the mucosa is of eminent importance for the defence of bacterial infection and maintenance of health. Apart from the adaptive immune mechanisms involving antigen presentation and $\mathrm{T}$ or $\mathrm{B}$ cell responses, innate mechanisms are needed for immediate responses. On the other hand, nutritional antigens and antigens from commensal bacteria must not lead to immune activation and inflammation but must be tolerated by the innate and adaptive immune system.

One molecule, which is able to modulate both the innate and adaptive immune system, is glycoprotein 96 (gp96, GRP94, Erp99, endoplasmin). Gp96 is an endoplasmic reticulum (ER) resident non-polymorphic heat shock protein (HSP) and is highly phylogenetically conserved. ${ }^{1}$ In humans, only one true gene locus has been mapped and was named tra- $1 .^{2}$ The gene product gp96 is one of the major peptide acceptors in the $\mathrm{ER}^{3}$ and interacts specifically with receptors, including CD91 ( $\alpha 2$-macroglobulin receptor $)^{4}$ and toll-likereceptors (TLR) 2 and 4 on the surface of professional antigen presenting cells (APCs). In BALB/c mice, gp96 was found to induce immunity against challenge with the tumour from which gp96 was originally purified..$^{5-7}$ Gp96 has been shown to bind peptides and this peptide binding property is responsible for induction of immunity. ${ }^{8}$ It is generally believed that gp96-peptide complexes are released into the extracellular compartment during stress and necrosis of cells and then bind to CD91 on APCs. This binding leads to internalisation of the gp96-peptide complex. ${ }^{49}$ The peptide is then presented by major histocompatibility complex (MHC) class I to T cells and immunity, ${ }^{10}$ or tolerance by induction of regulatory $\mathrm{T}$ cells, occurs. ${ }^{11}{ }^{12}$

The intestinal mucosa is exposed to a constant high level of antigens. Tolerance is achieved by downregulation of surface proteins involved in the reception or transmission of inflammatory signals on appropriate cells. ${ }^{13}$ On intestinal macrophages (IMACs) this refers to CDI4, ${ }^{14}$ TLR-2 and TLR-4, ${ }^{15}$ HLA-DR, ${ }^{16}$ and the integrins CDllb/CD18 and CDilc/CD18. ${ }^{17}{ }^{18}$ Also, the $\mathrm{T}$ cell costimulatory molecules CD80 (B7-1) and CD86 (B7-2) are downregulated in normal mucosa, ${ }^{16}$ indicating a low ability of IMACs to mediate immune responses. Functional data (for example, diminished oxidative burst activity ${ }^{16}{ }^{19}$ ) support the concept that IMACs are "desensitised" during differentiation. The inflamed mucosa however is populated by IMACs that are fully responsive to bacterial antigens. ${ }^{15}$ Expression of the $\mathrm{T}$ cell costimulatory molecules CD80 and CD86 furthermore indicates that IMACs have the ability to induce immune responses. ${ }^{16}$

\footnotetext{
Abbreviations: PBMNC, peripheral blood mononuclear cells; TLR, tolllike receptor; APC, antigen presenting cell; $B D H C$, benzidine dihydrochloride; CD, Crohn's disease; DAPI, 4',6-diamidino-2phenylindole; DNase, deoxyribonuclease; ER, endoplasmic reticulum; gp, glycoprotein; HSP, heat shock protein; IBD, intestinal bowel disease; iv mac, in vitro differentiated macrophages; IMACs, intestinal macrophages; LPMNCs, lamina propria mononuclear cells; MCS, multicellular spheroids; MHC, major histocompatibility complex; PBS, phosphate buffered saline; RT-PCR, reverse transcription-polymerase chain reaction; UC, ulcerative colitis
} 
An in vitro model for the differentiation of IMACs is coculture of monocytes with HT-29 spheroids. ${ }^{20}$ In this model monocytes are seeded onto spheroids of HT-29 cells and invade them followed by a differentiation process which results in a phenotype similar to IMACs in normal intestinal mucosa with lack of CD14, CD16, CD11b, and CDllc surface expression. $^{20}$

By subtractive hybridisation we found that gp96 was expressed in IMACs from normal mucosa but not in in vitro differentiated macrophages (iv mac). Therefore, we further investigated gp96 expression in normal and inflamed mucosa. The MCS model was used to study gp96 expression during differentiation of IMACs. Finally, we studied regulation of gp96 expression under inflammatory conditions in an animal model of colitis.

\section{MATERIALS AND METHODS Patients}

Tissue samples were obtained from intestinal mucosa of 14 patients with Crohn's disease (CD), five patients with ulcerative colitis (UC), three patients with diverticulitis, and 14 control patients with no intestinal inflammation who underwent surgery for other reasons (for example, colon cancer). The degree of inflammation was graded microscopically by determination of the inflammatory infiltrate of neutrophils, eosinophils, and lymphocytes: $0=$ no infiltration, $1=$ low degree of infiltration, 2 = severe infiltration. All control specimens were negative for inflammatory infiltrate and the CD, UC, and diverticulitis specimens all had a moderate to severe inflammatory infiltrate.

The study was approved by the University of Regensburg Ethics Committee.

\section{Isolation of human lamina propria mononuclear cells (LPMNCs)}

Surgical specimens from inflamed and normal mucosa were obtained by surgery. Specimens were obtained from the colon of patients with CD and UC after receiving informed consent. Control specimens were taken from patients who underwent surgery for other reasons (colonic carcinomas). On average, specimens were approximately $20 \mathrm{~cm}^{2}$ and we obtained about $1 \times 10^{6}$ IMACs. LPMNCs were isolated as described previously. ${ }^{13}$ In brief, mucosa was incubated in calcium and magnesium free Hank's balanced salt solution with $1 \mathrm{mmol} / \mathrm{l}$ ethylenediaminetetraacetic acid for 15 minutes at $37^{\circ} \mathrm{C}$ to remove intestinal epithelial cells. Intestinal epithelial cell depleted specimens were incubated for 30 minutes in $2 \mathrm{ml}$ phosphate buffered saline (PBS) with $1 \mathrm{mg} / \mathrm{ml}$ collagenase type I ( $=336 \mathrm{U} / \mathrm{ml}$ ), $0.3 \mathrm{mg} / \mathrm{ml}$ deoxyribonuclease (DNase I; Boehringer, Mannheim, Germany), and $0.2 \mathrm{mg} / \mathrm{ml}$ hyaluronidase without fetal calf serum at $37^{\circ} \mathrm{C}$. Cells were filtered by passing through a $70 \mu \mathrm{m}$ filter, washed in PBS, and finally submitted to Ficoll density gradient centrifugation for 20 minutes at $2000 \mathrm{rpm}(\approx 690 \mathrm{~g}$, without brake $)$ in a Heraeus centrifuge for isolation of mononuclear cells. The interphase was carefully removed and washed with PBS.

\section{Isolation and purification of IMACs}

IMACs in isolated LPMNCs were labelled with immunomagnetic MicroBeads, armed with CD33 antibody, and purified twice with the help of type LS separation columns (Miltenyi Biotec, Bergisch Gladbach, Germany), as described recently. ${ }^{13}$

\section{Generation of in vitro differentiated macrophages}

After leucapheresis, $1 \times 10^{6}$ monocytes were seeded in Teflon bags and cultured at $37^{\circ} \mathrm{C}$ with $7 \% \mathrm{CO}_{2}$. By addition of $2 \%$ human $\mathrm{AB}$ serum for seven days, macrophages were generated out of the monocytes. After incubation at $4^{\circ} \mathrm{C}$, cells detached from the Teflon bag.

\section{Subtractive hybridisation}

Subtractive hybridisation was performed, subtracting cDNA from iv mac from normal IMACs using the Clontech PCRSelect cDNA subtraction kit (Clontech, Palo Alto, California, USA), as previously described. ${ }^{16}{ }^{19}$ Two hybridisations were performed. In the first, excess cDNA from iv mac was added. Single strand molecules from normal IMACs were significantly enriched for differentially expressed sequences. The entire population of molecules was subjected to polymerase chain reaction (PCR) to amplify the desired differentially expressed sequences. Only molecules with two different adaptors were amplified exponentially. A secondary PCR amplification was performed using nested primers to further reduce any background PCR products and enrich for differentially expressed sequences.

With the clones obtained, a similarity search was performed at http://www.ncbi.nlm.nih.gov/index.html. The BLAST program was used to perform DNA database searches for sequence similarities.

\section{Affymetrix oligonucleotide array analysis}

Affymetrix GeneChip analysis was performed with help of the Kompetenzzentrum für fluoreszente Bioanatytik (KFB) in Regensburg (Germany). mRNA was isolated from IMACs from non-inflamed mucosa and from iv mac using polyT magnetic beads (Dynal, Oslo, Norway). In each case, RNA from three patients was pooled to avoid individual differences being responsible for variation.

Double stranded cDNA was synthesised by a Superscript II kit (Life Technologies, Karlsruhe, Germany) using T7oligo(dT) as primer. Biotin labelled cRNA was prepared by in vitro transcription reaction using an Enzo BioArray HighYield RNA Transcript Labelling Kit (Affymetrix, P/N 900182) based on the manufacturer's protocol. Biotin labelled cRNA was purified, fragmented and, after addition of Control Oligonucleotide B2 (3 nM) and 20× Eukaryotic Hybridisation Controls (bioB, bioC, bioD, cre), hybridised to Affymetrix HG-U133A GeneChips (Affymetrix, Santa Clara, California, USA), scanned on the Affymetrix array scanner, and data analysis performed using the Affymetrix statistical data analysis software, Affymetrix Microarray Suite (version $5.0)$.

\section{Reverse transcription-polymerase chain reaction (RT-} PCR) for gp96

Poly(A)-RNA was isolated by polyT magnetic beads (Dynal) from $\mathrm{CD}_{3} 3^{+}$cells according to the manufacturer's protocol. Oligonucleotides for gp96 PCR were chosen using the BTI software Gene Tool lite (version 1.0.0.1). For PCR, primers were used as follows: gp96 upstream, 5' GGG TGT GGT GGA CTC AGA TG 3'; gp96 downstream, 5' GTT GCC AGA CCA TCC GTA CT 3'. To test cDNAs for representation and full

Subtracted fragments from reference sequence X15187 (NCBI)

Precursor mRNA

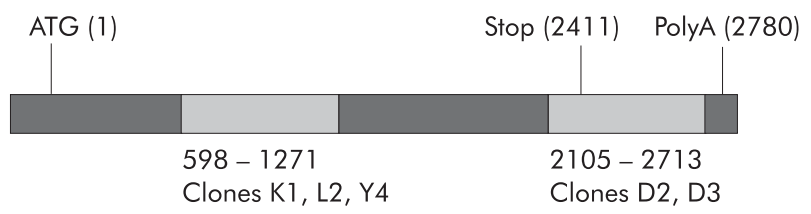

Figure 1 As a result of a similarity search, five of 76 clones obtained by subtractive hybridisation of intestinal macrophages and in vitro differentiated macrophages were identified as glycoprotein 96 (gp96). Thereby, two different parts of the cDNA were identified. Nucleotides 598-1271 were obtained three times and nucleotides 2105-2713 twice. 


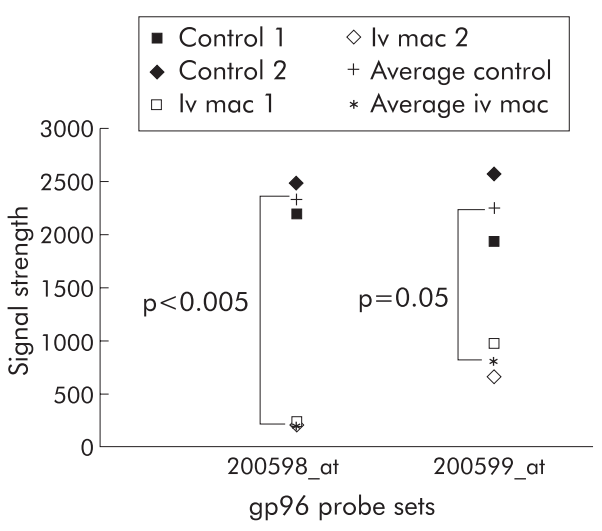

Figure 2 Affymetrix data confirmed the results of subtractive hybridisation. The original signal strength data are shown for the probe sets for intestinal macrophages (IMACs) and in vitro differentiated macrophages (iv mac). Each data point represents the original signal strength on the chip. The average is formed from the two data points for IMACs and iv mac. For probe set 200598_at, 11 -fold induction and for 2000599_at, threefold induction was observed. For statistical analysis the $t$ test was used. On average, induction of glycoprotein 96 mRNA expression in IMACs from normal mucosa compared with iv mac was sevenfold in the Affymetrix analysis.

length genes, RT-PCR with a 5' $\beta$-actin, 3' $\beta$-actin, GAPDH, $6 \mathrm{~K}$ clathrin, and $2 \mathrm{~K}$ clathrin primer set from the Gene Checker Kit (Invitrogen, Leek, Netherlands) was used.

\section{Generation of multicellular spheroids (MCS)}

MCS were generated according to the liquid overlay culture technique ${ }^{21}: 4 \times 10^{3}$ cells suspended in $0.2 \mathrm{ml}$ of medium/well

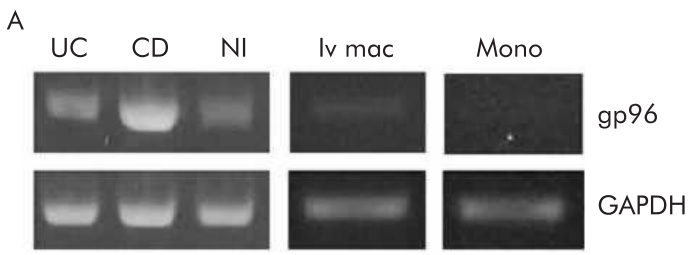

B

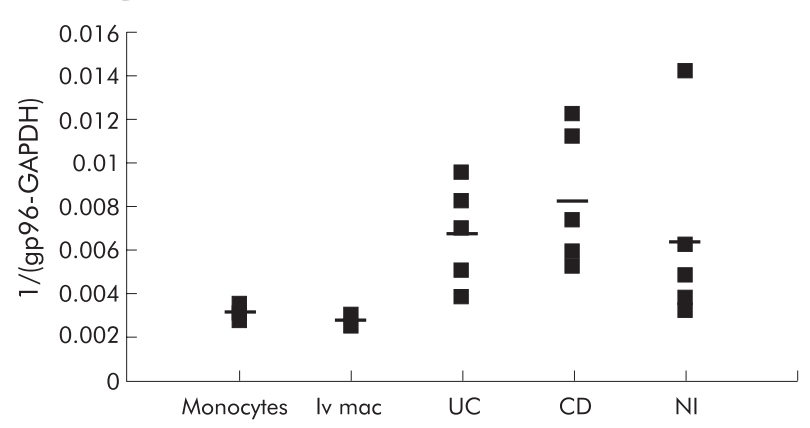

Figure 3 Glycoprotein 96 (gp96) mRNA expression in in vitro differentiated macrophages (iv mac), monocytes (mono), and in intestinal macrophages (IMACs) from patients with ulcerative colitis (UC), Crohn's disease (CD), and non-inflamed mucosa (NI). (A) Reverse transcription-polymerase chain reaction (RT-PCR) for gp96 and GAPDH, as indicated. Gp96 was detected in IMACs from UC, CD, and NI mucosa, but not in iv mac or monocytes. The figure is representative of 11 patients with $\mathrm{NI}$ mucosa, nine patients with $\mathrm{CD}$, six patients with $U C$, four donors for iv mac, and 11 monocyte donors. (B) Real time-PCR (TaqMan) for gp96. mRNA levels were higher in macrophages from patients with $C D, U C$, and $\mathrm{NI}$ mucosa than in monocytes and iv mac (monocytes $v$ UC, $\mathrm{p}<0.002$; monocytes $v \mathrm{CD}, \mathrm{p}<0.005$; monocytes $v$ $\mathrm{NI}, \mathrm{NS}$; iv mac $v$ UC, $\mathrm{p}<0.05$; iv mac $v \mathrm{CD}, \mathrm{p}<0.02$; iv mac $v \mathrm{NI}, \mathrm{NS}$ ). were seeded in agarose coated wells of 96 well plates and cultured under static conditions, as described. After seven days of culture, MCS had formed and were used for experiments. $^{20}$ MCS supernatants $(0.1 \mathrm{ml}$ each $)$ were replaced by freshly isolated monocytes in $0.1 \mathrm{ml}$ of medium supplemented with $2 \%$ human AB serum. Cocultures of MCS with monocytes were harvested after 24 hours, three days, and seven days for immunohistochemical analysis.

\section{Antibodies}

The following antibodies were used for immunohistochemical identification of gp96: rat anti-grp94 monoclonal antibody (clone 9G10, IgG2a; Stressgen Biotechnologies, Canada), rabbit anti-grp94 polyclonal antibody (Stressgen Biotechnologies), and biotin conjugated rabbit antirat and goat antirabbit secondary antibody (Sigma, Deisenhofen, Germany).

The following monoclonal antibodies were used for immunohistochemical identification of macrophages: mouse antihuman macrophage CD68 (clone KPl; Dako, Hamburg, Germany) and biotin conjugated goat antimouse secondary antibody (IgG, monoclonal; Sigma) for detection of mouse macrophages; and biotinylated rat anti-mac-3 (IgGl $\kappa$, monoclonal; Cederlane, Canada) and biotinylated rat antiF4/80 (IgG2a; Serotec, Germany). The monoclonal antibody mouse antihuman macrophage CD33 MicroBeads (Miltenyi Biotec, Bergisch Gladbach, Germany) was used for isolation of macrophages.

For immunofluorescence the following antibodies were used as secondary antibodies: Alexa Fluor 488 chicken antimouse IgG $(\mathrm{H}+\mathrm{L})$ (Molecular Probes Europe BV, the Netherlands) and Alexa Fluor 594 conjugated goat antirat IgG (Molecular Probes Europe BV).

\section{Immunohistochemistry/immunofluorescence}

For peroxidase staining, frozen sections were cut $(5 \mu \mathrm{m})$, air dried, and fixed in acetone. Slides were rehydrated with PBS ( $\mathrm{pH}$ 7.4) and incubated for 30 minutes with $0.3 \% \mathrm{H}_{2} \mathrm{O}_{2}$ in PBS to quench endogenous peroxidase. Slides were washed with PBS and incubated for 60 minutes with $1 \%$ bovine serum albumin (Biomol, Hamburg, Germany) in PBS (blocking buffer) with goat serum (dilution 1:100; Dako, Denmark) for CD68 staining and rabbit immunoglobulin fraction $(0.2 \mathrm{mg} / \mathrm{ml}$ total protein concentration; Dako) for gp96 staining.

To identify the cell types expressing gp96, an immunohistochemical procedure for sequential double antigen localisation was applied. ${ }^{22}$ Firstly, slides were incubated with either rat anti-gp96 (final concentration $5 \mu \mathrm{g} / \mathrm{ml}$ in blocking buffer) or mouse antihuman macrophage CD68 $(0.33 \mu \mathrm{g} / \mathrm{ml})$ primary antiserum in blocking buffer for 60 minutes. Secondly, slides were incubated for 30 minutes with 1:500 diluted biotin conjugated antirat or antimouse IgG secondary antibody in blocking buffer. After washing, tissue was incubated with a freshly prepared solution of Vector NovaRED (Vector, Burlingame, California, USA) containing $0.01 \%$ hydrogen peroxide. Slides were incubated for 30 minutes with $0.3 \%$ hydrogen peroxide in PBS buffer and incubated with the primary and secondary antibodies for the second staining. Sections were preincubated for eight minutes in $0.01 \%$ benzidine dihydrochloride (BDHC; Sigma) with 0.03\% sodium nitroprusside (Sigma). This was followed by incubation in the reaction medium $0.01 \%$ BDHC, $0.005 \%$ hydrogen peroxide, and $0.03 \%$ sodium nitroprusside) for blue staining. Staining was interrupted with water.

For immunofluorescence, all tissue specimens were fixed in $4 \%$ buffered formalin and embedded in paraffin. Specimens were dewaxed and pretreated in a water bath at $70^{\circ} \mathrm{C}$ for 40 minutes in $0.1 \%$ sodium citrate, blocked for 20 minutes with $20 \%$ goat serum at room temperature, and 

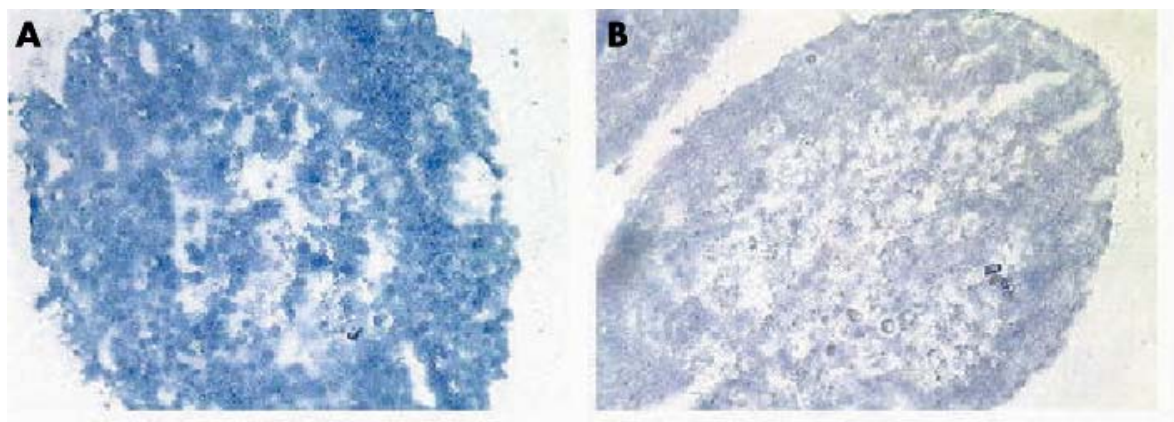

Figure 4 Expression of glycoprotein 96 (gp96) in HT-29 spheroids after 24 hours, three days, and seven days of coculture with monocytes. Antigen expression was determined by immunohistochemistry, as described in materials and methods. No gp96 expression was detected after 24 hours (A) or three days (B) of coculture. Gp96 expression was detected after seven days of coculture $(C, D)$. (E-G) Isotype controls for $A, B, C$, and $D$, respectively. Original magnification $\times 200$.
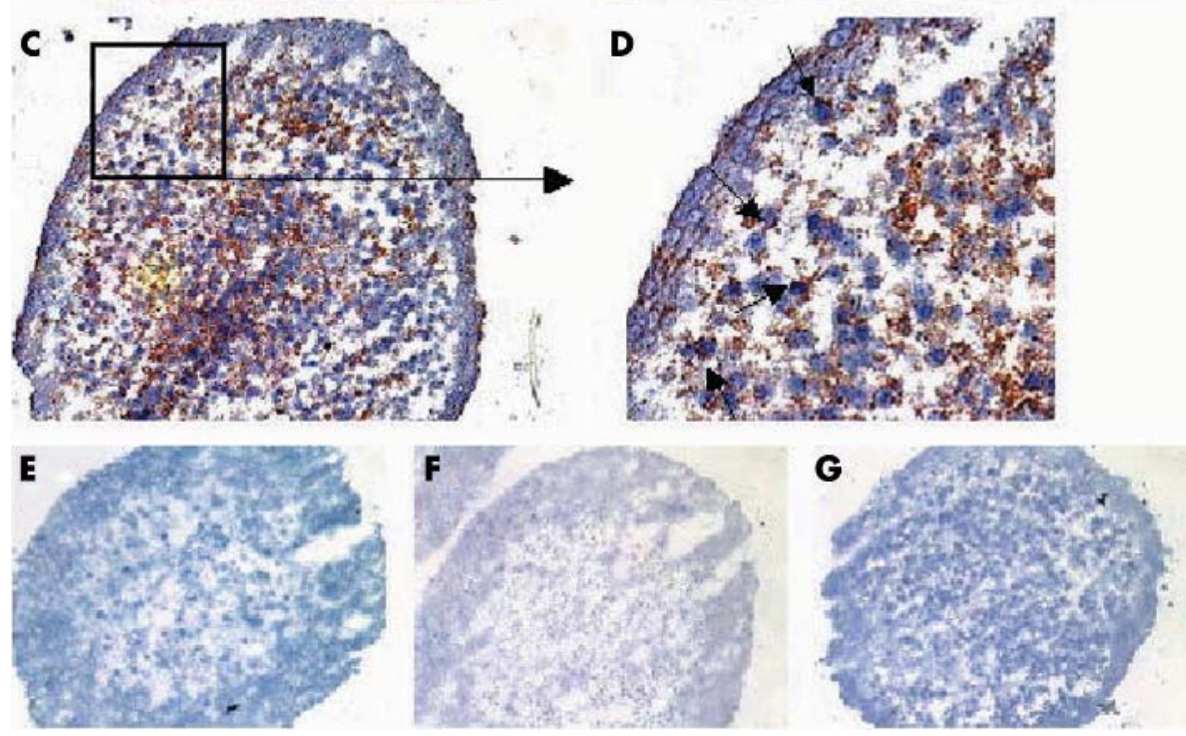

then incubated with the two primary antibodies at $4^{\circ} \mathrm{C}$ overnight (antibody dilution 1:100). Slides were washed in PBS and further incubated for one hour at $37^{\circ} \mathrm{C}$ with secondary antibodies (dilution 1:200) conjugated with fluorescein isothiocyanate or Texas red in the dark. Slides were washed and mounted in Vectashield mounting medium with $1.5 \mu \mathrm{g} / \mathrm{ml} \mathrm{4}$ ',6-diamidino-2-phenylindole (DAPI; Vector Laboratories). Microscopy was done with a Leitz DMRXE microscope, mostly with $400 \times$ magnification. Detailed information on magnification is given in the figure legends.

\section{CD4 ${ }^{+} \mathrm{CD} 2 \mathrm{~L}^{+} \mathrm{T}$ cell transfer model of colitis}

Splenic $\mathrm{CD}^{+} \mathrm{CD} 2 \mathrm{~L}^{+} \mathrm{T}$ cells from BALB/c mice were isolated as described previously with slight modifications. ${ }^{23}$ In brief, $\mathrm{CD}^{+} \mathrm{T}$ cells were purified from spleen mononuclear cells of healthy mice by negative depletion of other cell populations using anti-CD8, anti-MHC-II, anti-B220, and anti-CD1lb antibodies (purchased from Pharmingen, San Diego, California, USA) followed by antirat IgG immunomagnetic microbeads (Miltenyi Biotech, Bergisch Gladbach, Germany). $\mathrm{CD}^{+} \mathrm{T}$ cells were further separated by immunomagnetic beads into $\mathrm{CD}_{2} \mathrm{~L}^{+}$and $\mathrm{CD} 6 \mathrm{~L}^{-} \mathrm{T}$ cells. The former cells (purity $>95 \%$ ) showed high expression of CD45RB by FACS analysis. $\mathrm{CD}_{2} 2 \mathrm{~L}^{+} \mathrm{CD} 4^{+} \mathrm{T}$ cells $\left(0.25 \times 10^{6}\right)$ were resuspended in $200 \mu \mathrm{l}$ of sterile PBS and injected intraperitoneally into recipient CB-17 SCID mice. Colitis activity was monitored by weight change and histology analysis, as specified below.

\section{RESULTS}

Subtractive hybridisation, RT-PCR, and Affymetrix GeneChip analysis

To analyse whether specific proteins are induced during differentiation of IMACs, subtractive hybridisation of mRNA from iv mac and IMACs from normal mucosa was performed as described above. Evolving clones from this screening represented genes that are expressed in IMACs but not in iv mac.

Overall, 76 different clones were obtained. All cDNA fragments were cloned and sequenced. Some genes were obtained several times as sequenced clones contained different parts of the same gene. As genes obtained several times are considered more likely to be truly upregulated we focused on these clones. A search for the expressed sequence tag database showed that five of 76 subtracted products had $>99 \%$ homology with mRNA of gp96 (reference sequence in NCBI: X15187). We obtained two different fragments of gp96 (nucleotides 598-1271 and 2105-2713) from which one occurred three and the other two times (fig. 1).

To confirm the subtractive hybridisation data, we used Affymetrix GeneChip analysis. mRNA from three control patients and three donors for iv mac were pooled. mRNA in each case was hybridised onto two chips. For both probe sets for gp96 significantly higher mRNA expression was found in IMACs compared with iv mac. For each probe set we averaged the two chips. For one probe set nearly threefold and for the other 11 -fold higher gp96 mRNA expression in IMACs compared with iv mac was found (fig 2).

To further demonstrate the reliability of subtractive hybridisation and the Affymetrix GeneChip analysis, we performed RT-PCR for gp96 with mRNAs from CD-IMACs, UC-IMACs, and control-IMACs from monocytes and from iv mac. In macrophages from CD, UC, and non-inflamed mucosa, but not from blood monocytes, gp96 cDNA was amplified. Also, almost no gp96 cDNA was amplified in iv mac (fig 3A). The integrity of the mRNA was verified by the Gene Checker kit (fig 3A, only GAPDH shown). Additionally, 

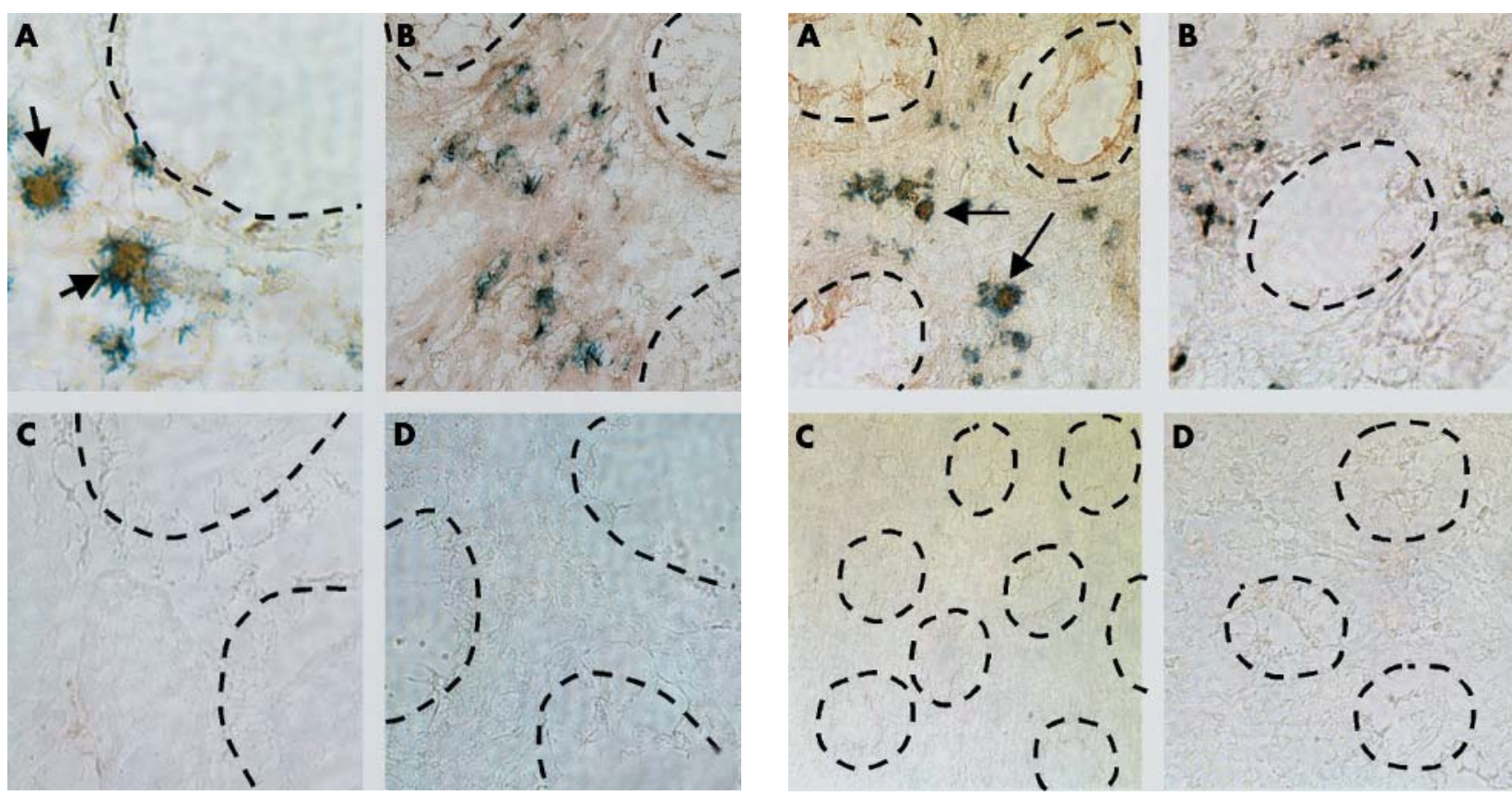

Figure 5 Detection of glycoprotein 96 (gp96) expression in intestinal macrophages of the intestinal mucosa with a monoclonal antibody.

Frozen sections were cut and fixed in acetone for peroxidase staining Gp96 was immunohistochemically detected in a first step with Vector NovaRED (red). In a second step the cellular marker for macrophages (CD68) was detected with benzidine dihydrochloride (BDHC) (dark blue, granular). Gp96 expression was detected in intestinal macrophages in non-inflamed mucosa (A). No gp96 expression was detected in intestinal macrophages in Crohn's disease (B). No NovaRED reaction product was detected using rat lgG2a isotype antibody in the first staining step. No $\mathrm{BDHC}$ reaction product was detected using mouse isotype control in the second staining step (C, D). The broken line indicates the crypt border. Original magnification $\times 400$.

we performed real time PCR (TaqMan) for gp96 with RNA from seven monocyte isolations, three cultures of iv mac, five UC patients, six CD patients, and five patients with no intestinal inflammation. As shown in fig 3B, there was no significant difference in mRNA levels from patients with UC, $\mathrm{CD}$, and no intestinal inflammation. However, there were significantly higher mRNA levels from monocytes and iv mac (monocytes $v$ UC, $\mathrm{p}<0.002$; monocytes $v \mathrm{CD}, \mathrm{p}<0.005$; monocytes $v$ no intestinal inflammation, NS; iv mac $v$ UC, $\mathrm{p}<0.05$; iv mac $v \mathrm{CD}, \mathrm{p}<0.02$; iv mac $v$ no intestinal inflammation, NS; $t$ test).

\section{Immunohistochemical analysis of gp96 expression in MCS}

Based on the results for mRNA expression, we assumed induction of gp96 during differentiation of IMACs. Therefore, we used the MCS model of IMACs differentiation ${ }^{21}$ to investigate whether gp96 is differentiation specific in IMACs.

Twenty four hours and three days after coculture of HT-29 cells with monocytes, no gp96 was detected in MCS (fig 4A, B). After seven days of coculture, gp96 was detected in high amounts in MCS in a pattern typical of invaded monocytes/ macrophages (fig 4C, D). This confirmed induction of gp96 during IMAC differentiation.

Gp96 protein expression in human intestinal mucosa Double labelling immunohistochemistry with specimens from nine patients without intestinal inflammation and seven patients with CD was performed. As shown in fig 5, gp96 was detected in IMACs from patients without intestinal inflammation (fig. 5A) but not in macrophages from patients

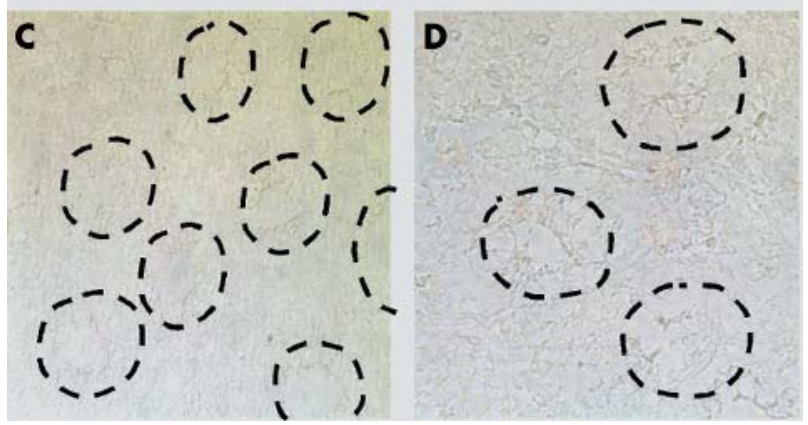

Figure 6 Detection of glycoprotein 96 (gp96) expression in macrophages of the intestinal mucosa with a polyclonal antibody. Frozen sections were cut and fixed in acetone for peroxidase staining Gp96 was immunohistochemical detected in a first step with Vector NovaRED (red). In a second step the cellular marker for macrophages (CD68) was detected with benzidine dihydrochloride (BDHC) (dark blue, granular). Gp96 expression was detected in intestinal macrophages in non-inflamed mucosa (A). No gp96 expression was detected in intestinal macrophages in CD (B). No NovaRED reaction product was detected using rabbit immunglobulins in the first staining step. No $\mathrm{BDHC}$ reaction product was detected using mouse isotype control in the second staining step (C, D). The broken line indicates the crypt border. Original magnification $\times 400$.

with CD (fig. 5B). To exclude the possibility that peptide fragments bound to gp96 mask the epitope for the monoclonal anti-gp96 antibody, additional immunohistochemistry with a polyclonal antibody was performed, revealing identical results (fig 6).

Identical results were obtained by immunofluorescence. As shown in fig 7A-C (arrows), gp96 was expressed in the vast majority of IMACs in normal non-inflamed mucosa. Gp96 negatively stained cells accumulated subepithelially and were preferentially detected close to crypts. Positively stained cells were mainly found in the deeper layers of the lamina propria. IMACs in the mucosa of patients with CD were mostly negative for gp96 (fig 7D-F, green arrows). Only a small number of IMACs stained positive for gp96 (fig 7D-F, white arrows). No gp96 protein expression was detected in other cells in the mucosa in all samples (figs 5-7). To determine whether the absence of gp96 found in CD-IMAC is specific for $\mathrm{CD}$ or whether it is an epiphenomenon of mucosal inflammation, we performed immunohistochemistry and immunofluorescence on sections of patients with diverticulitis and UC. As shown in fig 8, during diverticulitis (fig 8 AF) and UC (fig 8G-L), gp96 was detected in large amounts in IMACs similar to non-inflamed controls (figs 5-7, arrows). The distribution of gp96 positive IMACs in the lamina propria of patients with diverticulitis was the same as in patients without inflammation

Gp96 expression in the transfer colitis mouse model As the function of gp96 for mucosal tolerance and inflammation is difficult to study in humans, we further investigated whether gp96 expression and regulation is similar in an 

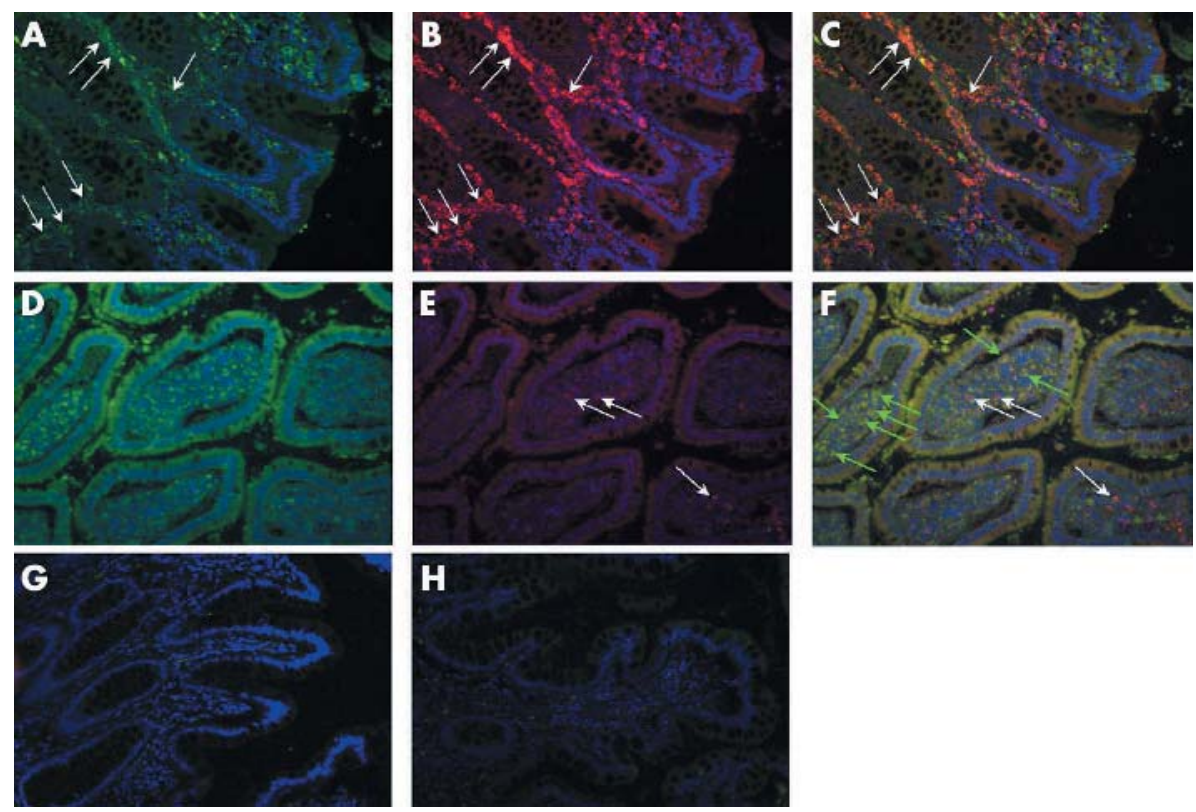

Figure 7 Immunofluorescence for glycoprotein 96 (gp96) expression in the intestinal mucosa of patients with no inflammation and with Crohn's disease (CD). Paraffin embedded sections were cut and gp96 was detected with a monoclonal antibody. In a second step, a secondary Alexa Fluor 594 conjugated goat antirat lgG antibody was used (red). CD68 was also detected with a monoclonal antibody and in a second step an Alexa Fluor 488 chicken antimouse $\lg G(\mathrm{H}+\mathrm{L})$ was used (green). Counterstain was done with 4', 6-diamidino-2-phenylindole (blue). In non-inflamed mucosa (A-C), most of the intestinal macrophages ( $A$, green, arrows) were gp96 positive ( $B$, red; $C$, merge, arrows). Only very few intestinal macrophages ( $D$, green) from $C D$ patients were gp96 positive ( $E$, red; $F$, merge, white arrows). No fluorescence was detected using rat lgG2a or mouse lgG1 isotype control $(G, H)$. Original magnification $\times 200$.

animal model of colitis. $\mathrm{CD} 4{ }^{+} \mathrm{CD} 62 \mathrm{~L}^{+}$cells were isolated from $\mathrm{BALB} / \mathrm{c}$ mice and transferred into SCID mice. After seven weeks SCID mice developed colitis and frozen sections from the intestine of seven mice with colitis and from five healthy mice without colitis were stained immunohistochemically to determine gp96 protein expression. As there is no typical general marker for macrophages in mice, we used two different antibodies (Mac-3 and F4/80) to detect macrophages in the intestinal mucosa. In mucosa from mice without colitis, gp96/Mac-3 positive macrophages were detected (fig 9A). In contrast, no gp96 was detected in the mucosa of mice with colitis (fig 9B, F). There were also no Mac-3 positive macrophages detected in the mucosa of mice with colitis (fig 9B). However, in Mac-3 positive macrophages that were located in lymph follicles of mice with colitis, gp96 protein was expressed (fig 9C, D). The majority of IMACs in healthy mice were negative for another macrophage marker, F4/80, which is assumed to be expressed in mature and activated macrophages. Only very few F4/80 positive IMACs were detected in the non-inflamed intestinal mucosa. These IMACs were negative for gp96 (fig 9E). In contrast, in the inflamed mucosa a high number F4/80 positive macrophages were detected which were gp96 negative (fig 9F). Taken together these data indicate that regulation of gp96 protein in this model of colitis is very similar to that found in human CD.

\section{DISCUSSION}

In the present study we demonstrated induction of gp96 expression during differentiation of IMACs at the mRNA and protein levels. Immunohistochemistry clearly showed gp96 expression in IMACs of the lamina propria from patients with normal non-inflamed mucosa, diverticulitis, and UC. Almost no gp96 protein expression could be found in the intestinal mucosa of patients with $\mathrm{CD}$, raising the possibility that absence of gp96 is involved in CD specific pathophysiology. In mice, gp96 was found to be expressed in mac-3 positive
IMACs in the non-inflamed mucosa but was absent in mature F4/80 positive IMACs in the inflamed mucosa of mice with colitis, indicating regulation similar to that found in humans.

Previously, gp96 expression was mainly studied in the context of malignant diseases, such as colorectal cancer ${ }^{24}$ or malignant mesothelioma. ${ }^{25}$ In both, gp96 expression was enhanced in tumour tissue compared with normal healthy tissue, which indicates that gp96 is involved in antigen presentation by peptide transfer to MHC class I. As a role for gp96 in antigen presentation and induction of tolerance is likely, the presence of this protein in IMACs is interesting. Our data indicate that gp96 is induced during specific differentiation of intestinal APCs. This points to a specific role for this protein in the mucosal immune system.

Gp96 is detected in high amounts in IMACs under conditions of mucosal tolerance and is decreased in CD, associated with loss of tolerance for its own bacterial flora. The role of gp96 during chronic inflammation has not been studied; in contrast, expression of Hsp 90, the cytosolic homologue of gp96, was investigated in patients with CD and UC. ${ }^{26}$ No difference in Hsp 90 expression between inflamed and non-inflamed mucosa was observed. ${ }^{26}$ However, Ludwig et al showed enhanced expression of Hsp 70 in epithelial cells in CD and UC, independent of the degree of inflammation. ${ }^{27}$ Peetermans et al demonstrated enhanced Hsp 60 expression in $\mathrm{B} 7$ positive mononuclear cells in the mucosa of patients with intestinal bowel disease (IBD) ${ }^{28}$ and enhanced Hsp 60 staining was reported in epithelial cells in UC. ${ }^{29}$ These investigations did not provide evidence for a role of these Hsps in chronic mucosal inflammation.

Our study is the first to investigate expression of gp96 in the intestinal mucosa in the context of IBD. In contrast with other studies which showed unchanged or enhanced expression of Hsps during IBD, we demonstrated here that gp96 was downregulated or absent in IMAC-CD. There are several reasons why gp96 may disappear in active $\mathrm{CD}$. The first 

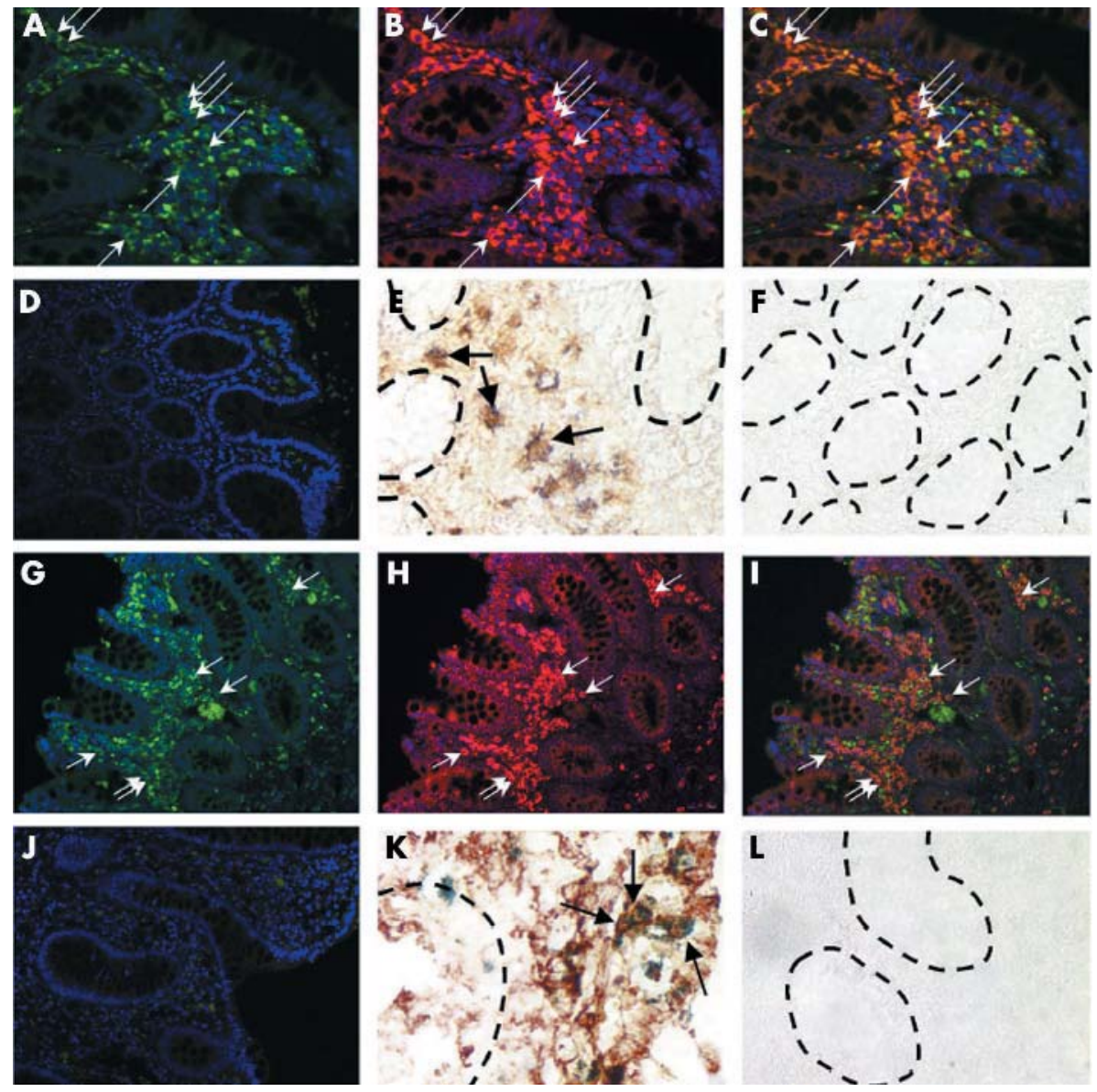

Figure 8 Immunohistochemical detection of glycoprotein 96 (gp96) on paraffin embedded and frozen sections from patients with diverticulitis (A-F) and ulcerative colitis (UC) (G-L). Paraffin embedded sections ( $A-D$ and $G-J$ ) were cut and gp96 was detected with a monoclonal antibody. In a second step, a secondary Alexa Fluor 594 conjugated goat antirat lgG antibody was used (red). CD68 was also detected with a monoclonal antibody, and in a second step an Alexa Fluor 488 chicken antimouse lgG $(\mathrm{H}+\mathrm{L})$ was used (green). Counterstain was done with 4', 6-diamidino-2-phenylindole (blue). Frozen sections $(E, F, K, L)$ were cut and fixed in acetone for peroxidase staining. Gp96 was immunohistochemically detected in a first step with Vector NovaRED (red). In a second step, the cellular marker for macrophages (CD68) was detected with benzidine dihydrochloride (BDHC) (dark blue, granular). In patients with diverticulitis (A-E) as well as in patients with UC (G-K), most intestinal macrophages were gp96 positive (arrows). No fluorescence or NovaRED reaction product was detected using rat lgG2a in the first staining step. No fluorescence or BDHC reaction product was detected using mouse isotype control in the second staining step (D, F, J, L). The broken line indicates the crypt border. Original magnification $A-D$ and G-J $\times 200 ; E, F, K, L \times 400$.

possibility is that gp96 protein is secreted by activated IMACs in inflamed CD mucosa in high amounts and is rapidly degraded. Our data from the Affymetrix GeneChip analysis showed that in IMACs from healthy non-inflamed mucosa, proteasome associated proteins were downregulated in comparison with iv mac (data not shown). In IMACs from inflamed mucosa, these genes can be upregulated again, so that protein degradation is enhanced and gp96 is degraded intracellularly. In qualitative PCR in both IMACs from normal and from inflamed mucosal tissue, gp96 mRNA was detected. Because of the low number of cells available after purification and a consecutive low amount of mRNA (under the level of detection), a high number (40) of cycles have to be done in PCR. Therefore, no conclusion about mRNA quantity can be made. Taqman data confirmed the results of subtractive hybridisation and Affymetrix GeneChip analysis which clearly showed enhanced gp96 expression in IMACs compared with monocytes and iv mac.

We showed that gp96 was induced in intestinal macrophages during differentiation of monocytes into an intestinal macrophage-like phenotype in the MCS model. During inflammation, monocytes invade the intestinal mucosa. Invading monocytes do not express gp96. This could explain, to some extent, why no gp96 protein was found in CD patients. However, mRNA expression was induced, as demonstrated. In addition, in UC, monocytes invade the lamina propria and finally express gp96 protein. Therefore, invasion of monocytes does not sufficiently explain the absence of gp96 protein in CD mucosa. An alternative explanation for downregulation of gp96 protein in CD intestinal macrophages could be the occurrence of post transcriptional or post translational regulation. This needs to be evaluated further.

As noted previously, downregulation of gp96 in IMACs of CD mucosa may play a role in loss of tolerance against luminal antigens. Chandawarkar et al found that immunisation with a 5-10-fold higher than optimal dose of gp96 downregulated antitumour immune responses. ${ }^{11}$ The authors showed recently that this effect was dependent on $\mathrm{CD}^{+} \mathrm{T}$ cells. ${ }^{12}$ These cells have to be further characterised but it is possible that $\mathrm{CD} 25^{+}$regulatory $\mathrm{T}$ cells are generated when gp96 is present in high amounts. It has been shown that gp96 cell surface expression in transgenic mice leads to a lupuslike autoimmune disease. Extracellular gp96 that has not bound processed peptides could serve as an endogenous APC activator. Chronic activation of APCs by gp96 could cause 


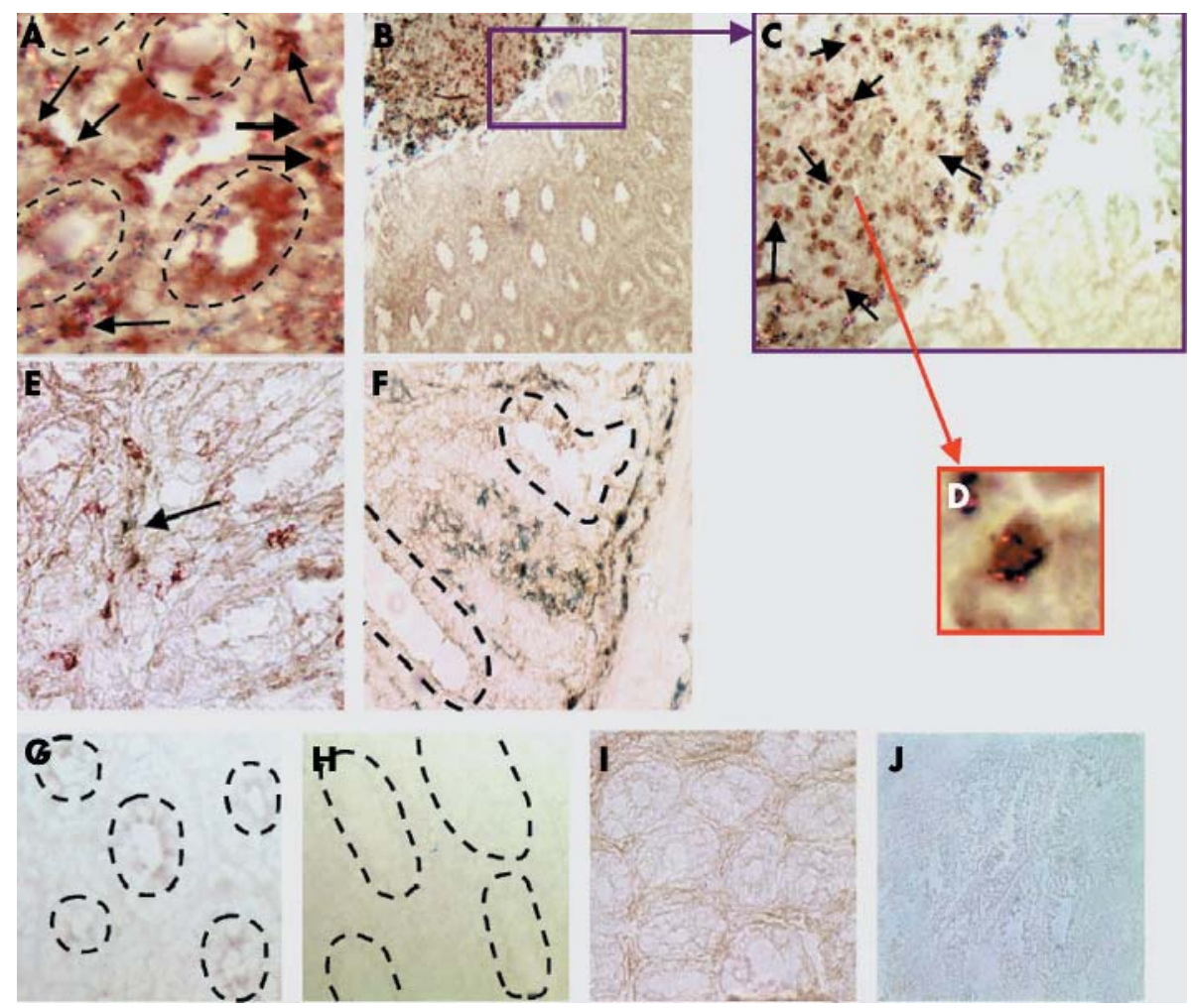

Figure 9 Immunohistochemical detection of glycoprotein 96 (gp96) from frozen sections of murine large intestine. Frozen sections were cut and fixed in acetone for peroxidase staining. Gp96 was immunohistochemically detected in a first step with Vector NovaRED (red). In a second step, the cellular marker for macrophages (mac-3 or F4/80) was detected with benzidine dihydrochloride (BDHC) (dark blue, granular). Gp96 was expressed in mac-3 positive macrophages (A) but was not detected in $\mathrm{F} 4 / 80$ positive macrophages (E) in non-inflamed mucosa. Gp96 was not expressed in mac-3 (B) or F4/80 (F) positive macrophages in the mucosa of mice with colitis. In lymphoid follicles, gp96 was detected in mac-3 positive macrophages (C, D). No NovaRED reaction product was detected using rat lgG2a in the first staining step. No BDHC reaction product was detected using rat isotype control in the second staining step (G-J). The broken line indicates the crypt border. Original magnification $\times 400$.

breakdown of tolerance. ${ }^{30}$ A similar mechanism could be responsible for maintaining the inflammation in the intestine during CD.

Recently, an immunomodulatory role for another related glycoprotein (HC gp39) was reported. In ex vivo assays, HC gp39 directed immune response was capable of suppressing cytotoxic $\mathrm{T}$ cell responses, indicating that the $\mathrm{HC}$ gp39 directed immune response in healthy individuals tends towards a regulatory phenotype. CD4+ T cell lines directed against HC gp39 expressed CD25, GITR, CTLA-4, and Foxp3 molecules and were capable of suppressing other immune responses. ${ }^{31}$ It could be speculated that gp96 has a similar effect in the intestinal mucosa. In fact, autoantibodies against gp96 can be detected in healthy mice. ${ }^{32}$ This interesting aspect warrants further study.

Very recently Doody et al demonstrated that gp96 can chaperone MHC II restricted epitopes for in vivo presentation to CD4+ T helper cells. CD4+ T cells can proliferate but are unable to express effector cytokines such as interferon $\gamma$ or interleukin $4 . .^{33}$

In conclusion, we have demonstrated that gp96 is induced during differentiation of monocytes into IMACs in humans and mice. We propose that gp96 functions as a tolerance mediating molecule. In CD mucosa and mouse colitis, gp96 is not detected. Further studies on the functional role of gp96 in the context of CD are under way.

\section{ACKNOWLEDGEMENTS}

This study was supported by the Deutsche Forschungsgemeinschaft (SFB 585) and the BMBF Kompetenznetz CED. We thank the surgeons and nurses of the surgery department for their excellent cooperation. In addition, we thank Marina Kreutz for kindly providing us the elutriated monocytes.

\section{Authors' affiliations}

K Schreiter, M Hausmann, T Spoettl, U G Strauch, J Schoelmerich,

H Herfarth, W Falk, G Rogler, Department of Internal Medicine I,

University of Regensburg, Regensburg, Germany

F Bataille, Department of Pathology, University of Regensburg,

Regensburg, Germany

Conflict of interest: None declared.

\section{REFERENCES}

1 Robert J, Menoret A, Basu S, et al. Phylogenetic conservation of the molecular and immunological properties of the chaperones gp96 and hsp70. Eur J Immunol 2001;31:186-95.

2 Maki RG, Old $\sqcup$, Srivastava PK. Human homologue of murine tumor rejection antigen gp96: 5 '-regulatory and coding regions and relationship to stressinduced proteins. Proc Nat Acad Sci U S A 1990;87:5658-62.

3 Lammert E, Arnold D, Nijenhuis $M$, et al. The endoplasmic reticulum-resident stress protein gp96 binds peptides translocated by TAP. Eur J Immunol 1997;27:923-7.

4 Binder RJ, Han DK, Srivastava PK. CD91: a receptor for heat shock protein gp96. Nat Immunol 2000;1:151-5

5 Srivastava PK, Deleo AB, Old $\amalg$. Tumor rejection antigens of chemically induced sarcomas of inbred mice. Proc Natl Acad Sci U S A 1986;83:3407-11.

6 Palladino MA Jr, Srivastava PK, Oettgen HF, et al. Expression of a shared tumor-specific antigen by two chemically induced BALB/c sarcomas. Cancer Res 1987;47:5074-9.

7 Srivastava PK, Chen YT, Old U. 5'-structural analysis of genes encoding polymorphic antigens of chemically induced tumors. Proc Natl Acad Sci U S A 1987:84:3807-11.

8 Li Z, Srivastava PK. Tumor rejection antigen gp96/grp94 is an ATPase: implications for protein folding and antigen presentation. Embo $J$ 1993;12:3143-51. 
9 Basu S, Binder RJ, Ramalingam T, et al. CD91 is a common receptor for heat shock proteins gp96, hsp90, hsp70, and calreticulin. Immunity 2001; 14:303-13.

10 Srivastava PK. Peptide-binding heat shock proteins in the endoplasmic reticulum: role in immune response to cancer and in antigen presentation. Adv Cancer Res 1993:62:153-77.

11 Chandawarkar RY, Wagh MS, Srivastava PK. The dual nature of specific immunological activity of tumor-derived gp96 preparations. J Exp Med 1999;189:1437-42.

12 Chandawarkar RY, Wagh MS, Kovalchin JT, et al. Immune modulation with high-dose heat-shock protein gp96: therapy of murine autoimmune diabetes and encephalomyelitis. Int Immunol 2004;16:615-24.

13 Rogler G, Hausmann M, Vogl D, et al. Isolation and phenotypic characterization of colonic macrophages. Clin Exp Immunol 1998;112:205-15.

14 Grimm MC, Pavli P, Van de Pol E, et al. Evidence for a CD14+ population of monocytes in inflammatory bowel disease mucosa-implications for pathogenesis. Clin Exp Immunol 1995;100:291-7.

15 Hausmann M, Kiessling S, Mestermann S, et al. Toll-like receptors 2 and 4 are up-regulated during intestinal inflammation. Gastroenterology 2002; 122:1987-2000.

16 Rogler G, Hausmann M, Spottl T, et al. T-cell co-stimulatory molecules are upregulated on intestinal macrophages from inflammatory bowel disease mucosa. Eur J Gastroenterol Hepatol 1999;11:1105-11.

17 Ingalls RR, Monks BG, Savedra R Jr, et al. CD1 1/CD18 and CD14 share a common lipid A signaling pathway. J Immunol 1998;161:5413-20.

18 Ingalls RR, Arnaout MA, Delude RL, et al. The CD11/CD18 integrins: characterization of three novel LPS signaling receptors. Prog Clin Biol Res 1998:397:107-17.

19 Hausmann M, Spottl T, Andus T, et al. Subtractive screening reveals upregulation of NADPH oxidase expression in Crohn's disease intestinal macrophages. Clin Exp Immunol 2001;125:48-55

20 Spottl T, Hausmann M, Kreutz $M$, et al. Monocyte differentiation in intestinelike macrophage phenotype induced by epithelial cells. J Leukoc Biol $2001 ; 70: 241-51$

21 Carlsson J, Yuhas JM, Liquid-overlay culture of cellular spheroids. Recent Results Cancer Res 1984;95:1-23.
22 Levey Al, Bolam JP, Rye DB, et al. A light and electron microscopic procedure for sequential double antigen localization using diaminobenzidine and benzidine dihydrochloride. J Histochem Cytochem 1986;34:1449-57.

23 Mudter J, Wirtz S, Galle PR, et al. A new model of chronic colitis in SCID mice induced by adoptive transfer of CD62L+CD4+ T cells: insights into the regulatory role of interleukin- 6 on apoptosis. Pathobiology 2002;70:170-6.

24 Heike M, Frenzel C, Meier D, et al. Expression of stress protein gp96, a tumor rejection antigen, in human colorectal cancer. Int J Cancer 2000:86:489-93.

25 Singhal S, Wiewrodt R, Malden LD, et al. Gene expression profiling of malignant mesothelioma. Clin Cancer Res 2003;9:3080-97.

26 Stahl M, Ludwig D, Fellermann K, et al. Intestinal expression of human heat shock protein 90 in patients with Crohn's disease and ulcerative colitis. Dig Dis Sci 1998;43:1079-87.

27 Ludwig D, Stahl M, Ibrahim ET, et al. Enhanced intestinal expression of heat shock protein 70 in patients with inflammatory bowel diseases. Dig Dis Sci 1999:44: 1440-7.

28 Peetermans WE, D’Haens GR, Ceuppens JL, et al. Mucosal expression by B7positive cells of the 60-kilodalton heat-shock protein in inflammatory bowel disease. Gastroenterology 1995;108:75-82.

29 Winrow VR, Mojdehi GM, Ryder SD, et al. Stress proteins in colorectal mucosa. Enhanced expression in ulcerative colitis. Dig Dis Sci 1993;38:1994-2000

30 Liu B, Dai J, Zheng H, et al. Cell surface expression of an endoplasmic reticulum resident heat shock protein gp96 triggers MyD88-dependent systemic autoimmune diseases. Proc Natl Acad Sci U S A 2003; 100:15824-9

31 Toes $\mathbf{R}$, van Bilsen J, van Dongen $\mathrm{H}$, et al. Cellular-based immune modulation in arthritis. Arthritis Res Ther 2004:6:18.

32 Menoret A, Chandawarkar RY, Srivastava PK. Natural autoantibodies against heat-shock proteins hsp70 and gp96: implications for immunotherapy using heat-shock proteins. Immunology 2000;101:364-70.

33 Doody AD, Kovalchin JT, Mihalyo MA, et al. Glycoprotein 96 can chaperone both MHC class I- and class II-restricted epitopes for in vivo presentation, but selectively primes CD8+ T cell effector function. J Immunol 2004; 172:6087-92.

\section{EDITOR'S QUIZ: GI SNAPSHOT}

\section{An unusual cause of rectal bleeding in a young woman}

\section{Clinical presentation}

A 24 year old woman presented with bloody rectal discharge of three months' duration. She had a medical history of herpes progenitalis and candida vaginitis. On examination, there was no tenderness in the abdomen. Routine stool culture and blood tests were negative. Colonoscopy with indigo carmine dye revealed friable and erythematous nodular mucosa in the lower rectum (fig 1).

\section{Question}

What is the diagnosis?

See page 949 for answer

This case is submitted by:

\section{A Hokama, F Kinjo, T Makishi, R Tomiyama, K Kishimoto, T Hirata N Kinjo, A Saito}

First Department of Internal Medicine, University of the Ryukyus, Okinawa,

K Takagi, T Arakak

Nishinjo Hospital, Okinawa, Japan

Correspondence to: Dr A Hokama, First Department of Internal Medicine, University of the Ryukyus, 207 Uehara, Nishihara, Okinawa 903-0215, Japan; hokama-a@med.u-ryukyu.ac.jp

doi: 10.1136/gut.2004.061424
Robin Spiller, Editor

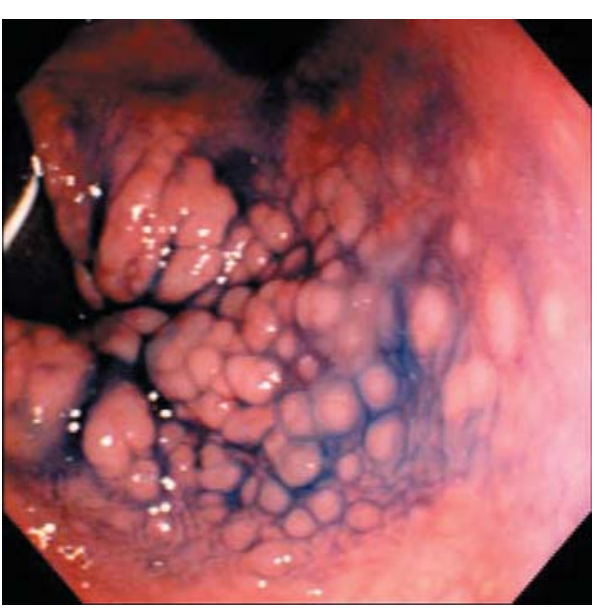

Figure 1 Colonoscopy with indigo carmine dye of the lower rectum. 